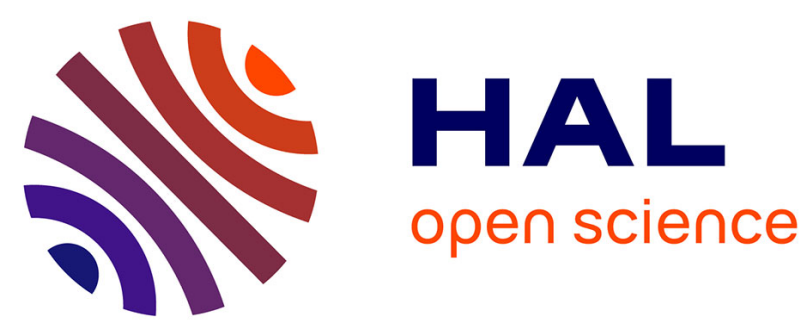

\title{
Experimental Implementation of Optical-CDMA for Medical Extra-WBAN Links
}

\author{
Jahid Hasan, Mohammad Ali Khalighi, Bastien Béchadergue
}

\section{To cite this version:}

Jahid Hasan, Mohammad Ali Khalighi, Bastien Béchadergue. Experimental Implementation of Optical-CDMA for Medical Extra-WBAN Links. 12th IEEE/IET International Symposium on Communication Systems, Networks and Digital Signal Processing (CSNDSP), Jul 2020, Porto, Portugal.

hal-02961319

\section{HAL Id: hal-02961319 \\ https://hal.science/hal-02961319}

Submitted on 8 Oct 2020

HAL is a multi-disciplinary open access archive for the deposit and dissemination of scientific research documents, whether they are published or not. The documents may come from teaching and research institutions in France or abroad, or from public or private research centers.
L'archive ouverte pluridisciplinaire HAL, est destinée au dépôt et à la diffusion de documents scientifiques de niveau recherche, publiés ou non, émanant des établissements d'enseignement et de recherche français ou étrangers, des laboratoires publics ou privés. 


\section{Experimental Implementation of Optical-CDMA for Medical Extra-WBAN Links}

\author{
$1^{\text {st }}$ Md Jahid Hasan \\ Aix-Marseille University, CNRS, \\ Centrale Marseille, Institut Fresnel \\ Marseille, France \\ Oldecomm SAS \\ Vélizy-Villacoublay, France \\ jahid.hasan@fresnel.fr
}

\author{
$2^{\text {nd }}$ Mohammad Ali Khalighi \\ Aix-Marseille University, CNRS, \\ Centrale Marseille, Institut Fresnel \\ Marseille, France \\ ali.khalighi@fresnel.fr
}

\author{
$3^{\text {rd }}$ Bastien Béchadergue \\ Oldecomm SAS \\ Vélizy-Villacoublay, France \\ bastien.bechadergue@oledcomm.net
}

\begin{abstract}
We consider the use of optical wireless technology based on infrared signals for multi-user wireless body-area networks (WBANs) in medical applications. For the case of uplink extra-WBAN signal transmission, we propose a simple optical code-division multiple access (O-CDMA) signaling scheme to manage the multiple-access channel. We experimentally evaluate the link performance in terms of bit- and frame-error-rates by implementing the proposed scheme on the programmable logic, and show the efficiency of this approach in dealing with multi-user interference, as well as its low-complexity.
\end{abstract}

Index Terms-Body area networks, infrared link, implementation of WBAN, optical wireless communications, optical codedivision multiple access, WBAN prototypes.

\section{INTRODUCTION}

The recent development of integrated wearable health monitoring sensors and the internet have already enabled e-health solutions to monitor patients from remote locations [1]. These health sensors communicate through the use of wireless bodyarea networks (WBANs), composed of intra- and extra-WBAN links. Intra-WBAN links allow transmitting data from different sensors to a coordinator node $(\mathrm{CN})$, placed on the patient's body [2]. Then, the $\mathrm{CN}$ sends the collected data to an access point (AP), via an extra-WBAN link [3]-[5].

Existing WBAN solutions based on radio-frequency (RF) technology suffer from electromagnetic interference (EMI), due to using the unlicensed band. Therefore, optical wireless communications (OWCs) using visible-light or eye-safe infrared (IR) have become an interesting solution, in particular, thanks to their inherent security features and the license-free noncongested optical spectrum [6].

In practical use cases of medical WBANs, several patients may need to transmit their vital signs simultaneously through a shared network, which can be enabled by employing multiple access (MA) schemes. The optical code-division MA (OCDMA) technique was proposed for medical WBANs in [7], [8], due to its robustness, low implementation complexity, low data-rate requirement (less than $100 \mathrm{Kbps}$ ), and enhanced security features. Unlike time-division MA, O-CDMA allows asynchronous data transmission by several users by assigning an optical orthogonal code (OOC) to each user. Note that frequency-division-based MA techniques suffer from implementation complexity and relatively low energy efficiency. This is crucial for extra-WBANs as the $\mathrm{CN}$ requires a relatively long battery lifetime (typically one week [9]).

O-CDMA was first introduced for use in optical fiber communications in [10]-[12]. There have been also several theoretical studies to investigate the performance of O-CDMA for OWC in indoor environments [13]-[16]. Different receiver $(\mathrm{Rx})$ structure of O-CDMA for wireless local area networks was studied from a digital design perspective in [17], and experimentally investigated in [18]. Also, in the WBAN context, the effect of patients' mobility on the performance of O-CDMA inside a hospital room was analyzed through simulations for intra- and extra-WBANs [7], [8].

To the best of our knowledge, the practical design and implementation of O-CDMA for medical WBANs have not been explored in detail so far. Indeed, O-CDMA implementation can be challenging, as its performance depends on the quality of the algorithms developed for synchronization between a transmitter (Tx) and a Rx [19]. The performance may also be severely impaired by MA interference (MAI) due to the well-known near-far problem in asynchronous mode [20], [21].

This paper investigates the implementation of an O-CDMA system for extra-WBAN in medical applications based on IR signals. Since extra-WBAN links are mainly used for transmitting data from a $\mathrm{CN}$ placed on the patient body to an AP, we focus here on the uplink. First, we compare different possible O-CDMA Rx architecture to select the most suitable one in our application, given the requirements of low implementation complexity, and the constraints imposed by near-far problem and the MAI effect. Then, we implement the selected O-CDMA $\mathrm{Rx}$ structure on a field-programmable gate array (FPGA) and propose an analog front-end (AFE) for the transmission and reception of the optical signals. Finally, we evaluate the performance of the implemented system in managing the MAI in terms of bit-error-rate (BER) and frame-error-rate (FER). We show that our Rx structure can operate with a BER lower than $10^{-3}$ without any error correcting algorithm up to $20 \mathrm{~cm}$ for the average $16 \mathrm{~mW}$ transmit power.

The remainder of the paper is organized as follows. In Section II, we describe O-CDMA based systems, including system architecture and digital design concepts. Next, Section III presents the implementation details of the FPGA prototypes. Experimental results are presented in Section IV. Lastly, the 
main conclusions of the work are drawn in Section V.

\section{SYSTEM DESCRIPTION}

\section{A. General Assumption}

We consider the case of four patients in a hospital ward, sending their health monitoring data via their respective $\mathrm{CN}$ to a single AP placed in the center of the ceiling. Each $\mathrm{CN}$, placed on the patient's shoulder to minimize the probability of line-of-sight (LOS) blocking, transmits its OOC-coded data asynchronously to the AP. In other words, transmission takes place without any synchronization between different CNs.

We assume that the data-rate requirement of each $\mathrm{CN}$ is less than $100 \mathrm{Kbps}$ [3], [5]. Given this relatively low data-rate, we use the simple on-off-keying (OOK) modulation. Also, we assume that there is always a LOS link between the Tx and the $\mathrm{Rx}$, and hence, neglect the effect of multiple reflections.

\section{B. General Principles of OOC-Based O-CDMA}

In O-CDMA signaling scheme, at the Tx side, each user (i.e., $\mathrm{CN}$ ) is at first assigned a signature code (SC) such as an OOC. Each bit from the user data stream is multiplied by this OOC and transmitted through the optical channel. The signals transmitted by all users are received on the Rx's PD. To recover the data of a specific user, the received signals are at first correlated with the corresponding assigned OOC. The output of each correlator is then compared with a predefined threshold $C_{\mathrm{Th}}$. The resulting signal is then demodulated to extract the transmitted bits of each user.

An OOC is a sequence of binary values called chips, denoted by $\operatorname{OOC}\left(L, W, \zeta_{a}, \zeta_{b}\right)$, where $L$ is the OOC length, $W$ is the number of ones in the sequence called weight, and $\zeta_{a}$ and $\zeta_{b}$ stand for the auto- and cross-correlation constraints [22]. By definition, strict orthogonality between such binary sequences can be achieved when $\zeta_{a}=\zeta_{b}=0$. However, our signaling here is based on intensity modulation and direct detection that relies on real and positive signals. Therefore, OOCs satisfy instead $\zeta_{a}=\zeta_{b}=1$, which provides the best trade-off between $\zeta_{a}$ and $\zeta_{b}$. For $\operatorname{OOC}(L, W, 1,1)$ codes, the maximum number of users $N$ is upper bounded by [22]:

$$
N \leq \frac{L-1}{W(W-1)}
$$

In an O-CDMA system with OOC codes, error-free data recovery is possible despite $\mathrm{MAI}$, if $C_{\mathrm{Th}}$ and $W$ satisfy the following conditions [10], [22]:

$$
C_{\mathrm{Th}}>N-1 \text { and } W>N-1 .
$$

\section{O-CDMA Rx Structure}

O-CDMA's performance highly depends on the type of Rx structure used to detect and retrieve data. The three most viable $\mathrm{Rx}$ structures for OWCs are correlator, chip-level detector (CLD), and correlator with a hard-limiter (CHL) [17].

A correlator or correlation $\mathrm{Rx}$ is the simplest $\mathrm{Rx}$ structure, see Fig. 1(a). It consists of a low pass filter and an analog-todigital (A/D) converter with a sampling rate at least equal to the chip time. The sampled chips are correlated with the desired user's OOC code, and the output is then compared with an

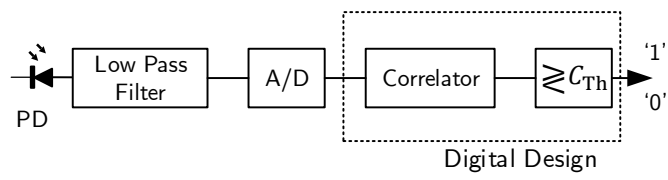

(a)

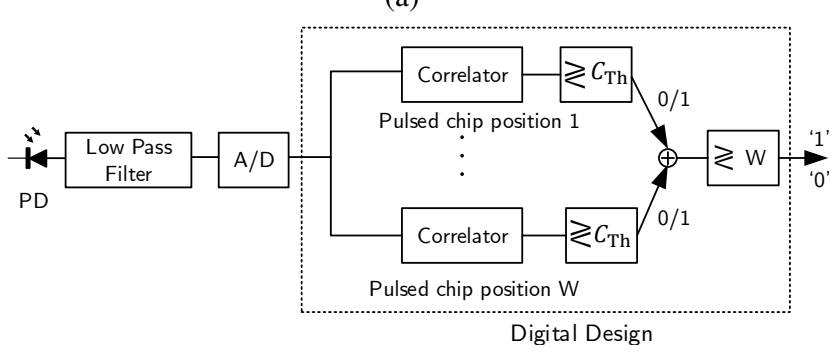

(b)

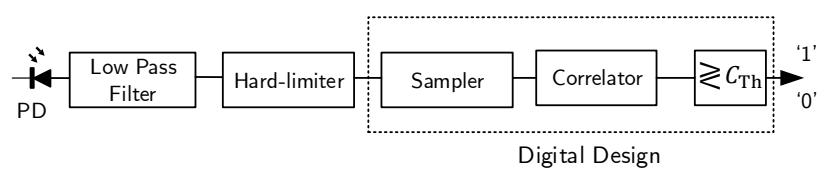

(c)

Fig. 1: Different types of Rx structures (a) Correlation Rx: Based on a simple correlator, (b) CLD: The correlation is done based on the received power of each chip, (c) CHL: An HL is added before a simple correlator.

optimal threshold $C_{\mathrm{Th}}$ to detect a bit ' 0 ' or ' 1 '. Correlation $\mathrm{Rx}$ suffers from the MAI and the near-far problem [17].

The CLD is based on the power of each chip collected by the PD, which is compared to an optimal threshold [12]. It also consists of a filter and an A/D converter as shown in Fig. 1(b). Each arm in the figure is used to detect one pulsed (i.e., ON) chip. Finally, all the values from each arm are added and compared with the weight $W$ to detect the transmit bits. The performance of the CLD is better than that of the correlation Rx [12]. However, it also suffers from near-far problem [12].

In a CHL Rx, a hard-limiter (HL) is added before the correlator [17], which clips the received signals at a defined level of threshold voltage, and hence converts the signals into binary sequences. Sampling takes place right after the HL with a locally generated clock with frequency at least equal to the chip rate, see Fig. 1(c). Therefore, the sampler of a CHL Rx does not require an A/D and can be directly implemented on an FPGA, which significantly reduces its implementation complexity. The main interest of CHL is that it minimizes the MAI effect by restricting the voltage at the same level for all users [23], [24]. Furthermore, the near-far problem is reduced because the defined threshold voltage ensures reception of balanced signals from near and far users. Here, due to its lower implementation complexity and its resilience to MAI and the near-far problem, we consider to use CHL.

\section{Digital Design Concept on Tx/Rx Synchronization}

As stated in Section II-A, CNs transmit their OOC-coded data to the AP without any synchronization between them. However, at the AP side, it is crucial to have bit-level synchronization between the corresponding Tx-Rx pairs in order to recover the data of each user properly. This bit-level syn- 


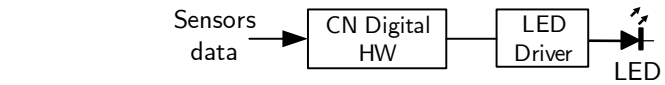

(a) $\mathrm{CN}$

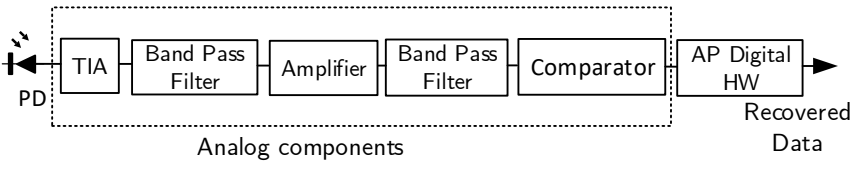

(b) AP

Fig. 2: Overall architectures of (a) a $\mathrm{CN}(\mathrm{Tx})$ and (b) the AP (Rx) with a focus on the main AFE blocks.

chronization relies on mechanisms implemented on both the Tx and the Rx sides as explained in the following.

1) $T x$ : On the $T x$ side, training patterns are added before transmitting OOC-coded information bits, enabling the $\mathrm{Rx}$ to synchronize its code with the corresponding $\mathrm{Tx}$, provided that the Rx has full knowledge of the training pattern sent. The length of the training pattern depends on the type of synchronization algorithm used by the Rx [17].

2) $R x$ : The synchronization process in an O-CDMA Rx is realized by an acquisition and tracking (AT) circuit, as in RFbased CDMA Rxs [25], [26]. Initial phase synchronization is done at the acquisition stage with a precision better than half of the chip duration $T_{c}$. Three kinds of acquisition algorithms are proposed for use in OWC, namely simple serial search, multiple shift, and matched filter [17]. The tracking circuit is the second step of the synchronization process. It is used for fine tuning, based on the generation of half-chip early and late replicas of the data. Correlation is then done with each replica and compared with a threshold $C_{\mathrm{Th}}$ to ensure synchronization.

In practice, it is shown that the matched filter algorithm is preferable to the other approaches as it requires a smaller number of training patterns and a shorter mean acquisition time [17]. Thus, we consider this solution in our design.

\section{IMPLEMENTATION}

\section{A. Overall Architecture of O-CDMA System}

As explained in Section II-A, our O-CDMA based extraWBAN system consists of four patients, each with a $\mathrm{CN}$ attached on the shoulder, and an AP at the center of the room ceiling. The architectures of a $\mathrm{CN}$ and the $\mathrm{AP}$ are shown in Figs. 2(a) and 2(b), respectively. Both the $\mathrm{CN}$ and $\mathrm{AP}$ are composed of two main parts: the digital hardware (HW) for generation or recovery of the O-CDMA data stream, and the AFE for transmission or reception of the optical signals. In the subsequent subsections, we discuss the implementation details of the AFE and the digital HWs.

\section{B. AFE Design}

On the $\mathrm{CN}$ side, the role of the AFE is to transmit the OOC-coded data stream generated by the digital HW circuitry through the use of a light-emitting diode (LED). Since this data stream is a binary sequence, we use a simple LED driver based on a MOSFET switch, see Fig. 2(a).

On the AP side, as represented in Fig. 2(b), the AFE consists of a PD followed by a trans-impedance amplifier (TIA) to convert the received photo-current into a voltage. The resulting

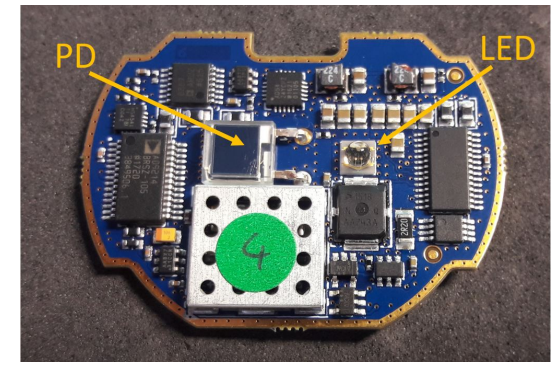

Fig. 3: The off-the-shelf AFE board for IR optical transmission and reception.

signal is then passed through a first band-pass filter to filter out noises generated by the ambient light and the TIA (i.e., the thermal noise), followed by a voltage amplifier and a second band-pass filter to center the signal around $0 \mathrm{~V}$. Finally, we employ a comparator as a zero-crossing detector, rather than a conventional HL, in order to boost the positive part of the signal to a fixed level (here, to $3.2 \mathrm{~V}$ ) while setting the negative part of the signal to $0 \mathrm{~V}$. This way, the AFE output signal meets the input/output standard LVCMOS $3.2 \mathrm{~V}$ of our FPGA board.

In practice, both the transmission and reception AFEs are implemented on a single board, as represented on Fig. 3. The LED operates at $940 \mathrm{~nm}$ with a semi-angle at half power of $60^{\circ}$. The Tx $3 \mathrm{~dB}$ modulation bandwidth (BW) is $9 \mathrm{MHz}$. On the $\mathrm{Rx}$ side, the $3 \mathrm{~dB} \mathrm{BW}$ is $15 \mathrm{MHz}$ and the overall $\mathrm{Rx}$ gain considering TIA and amplifier is $62 \mathrm{~dB}$. The PD has a $26.4 \mathrm{~mm}^{2}$ active surface, a $0.6 \mathrm{~A} / \mathrm{W}$ sensitivity at $940 \mathrm{~nm}$ and a full angle at half-sensitivity of $120^{\circ}$.

\section{CN Digital HW Design}

In the $\mathrm{CN}$ digital $\mathrm{HW}$, the serial data stream is encoded with the corresponding OOC code. Here, without loss of generality, we consider $L=8$ data bits per transmitted packet in addition to $L_{p}=3$ training bits (which are set to one) as preamble for synchronization purpose. The corresponding block diagram is shown in Fig. 4.

First, the serial data stream is passed through a serial-toparallel shift register ("SIPO" block in Fig. 4) in order to form the payload of the O-CDMA data frame. The SIPO block is followed by a dual clock first-input first-output (FIFO) memory block. Note that in digital system design, FIFOs are used to transfer data between two different clock domains [27]. Here, the FIFO is used to transfer data from the local clock domain (i.e., clock for sensors data) to the chip clock domain.

Each data bit then has to be multiplied by the OOC. By considering a maximum of four users, we deduce from Equations (1) and (2) that the OOC length has to be at least $F=49$. Since the input data rate is assumed to be $100 \mathrm{Kbps}$, see Section II, the chip clock rate at which the OOC-coded data have to be sent is set to $5 \mathrm{MHz}$. In practice, the multiplication with the $\mathrm{SC}$ is done by an AND logic gate. Therefore, each bit of the payload is first duplicated $F$ times by the "Reshape" block and then passed through an AND gate to apply the OOC. Note that the training bits are also OOC-coded to avoid MAI from other users (see Fig. 4). These $L_{p}$ sequences are added to the beginning of the frame by the aid of a multiplexer (the "MUX" block), controlled by the "Control Sequence" block. Finally, a 


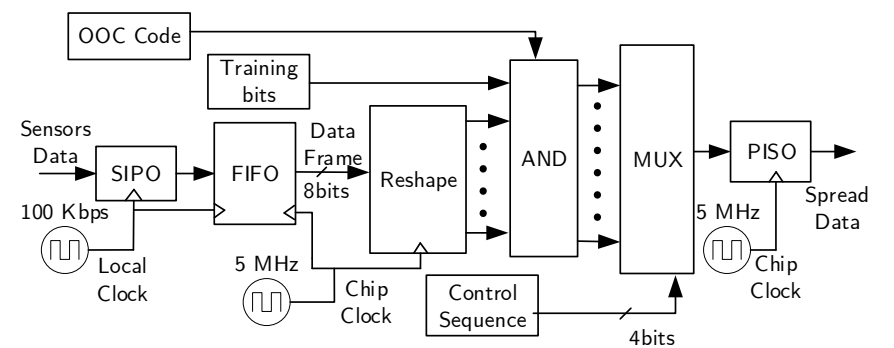

Fig. 4: Conceptual block diagram of $\mathrm{CN}$ digital HW based on $\mathrm{O}$ CDMA for extra-WBAN links. SIPO, PISO, and MUX stand for serialin parallel-out, parallel-in serial-out shift register, and multiplexer, respectively.

parallel-to-serial shift register ("PISO" block in Fig. 4) converts the OOC-coded data frames to serial spread data, which are then transferred to the Tx AFE.

\section{AP Digital HW Design}

The architecture of the AP digital HW for detecting the data of a given user is represented in Fig. 5. The same architecture is used for every user. As it can be seen, the data are first sampled ("Sampler" block in Fig. 5). The sampling process is done in the digital domain with a locally generated sampling clock $C K(t)$ of frequency $5 \mathrm{MHz}$. From a digital design point of view, synchronization failure is very common when sampling an asynchronous input with respect to a clock, that is when the asynchronous input (i.e., spread data) changes during the set up time of the clock [28]. To deal with this problem, we used three cascaded flip-flops (FFs) inside the sampler.

After sampling, at first, acquisition is performed in the "AV" block. This latter searches the training patterns by matched filter algorithm, as described in [17], and obtains initial time reference of the received spread data with a precision better than half of the chip duration $T_{c}$. Then the received spread data is sent to the verification stage in the "AV" block to detect false alarms (due to MAI and/or noise) by comparing with a predefined threshold. If a false alarm is detected, then the "AV" block starts performing acquisition again. Otherwise, the Acquisition Performed flag is set to high (see Fig. 5). This latter, in fact, activates acquisition or tracking process of the de-multiplexer ("DEMUX" block).

Afterwards, the tracking circuit starts more precise synchronization using the so-called early-late technique. To do this, the "ST" block generates early and late versions of the data, sampled by the shifted clocks $C K\left(t-T_{c} / 2\right)$ and $C K\left(t+T_{c} / 2\right)$, respectively. These replicas of the data are correlated with the OOC code, and the outputs of the correlators are then compared with the main data (i.e. the data that is sampled with the clock $C K(t)$ ) to obtain tracked spreading data. Meanwhile, if the outputs of the correlators are less than the threshold $C_{\mathrm{Th}}$, a synchronization error signal is sent to the "AV" block to start a new acquisition.

\section{EXPERIMENTAL RESULTS}

To implement our O-CDMA digital HW, we consider the case of two CNs (Txs), transmitting their data simultaneously to a single AP $(\mathrm{Rx})$. We have assigned the codewords $\{0,1,4,14\}$ and $\{0,2,7,26\}$ to the $\mathrm{CNs}$, where each number represents

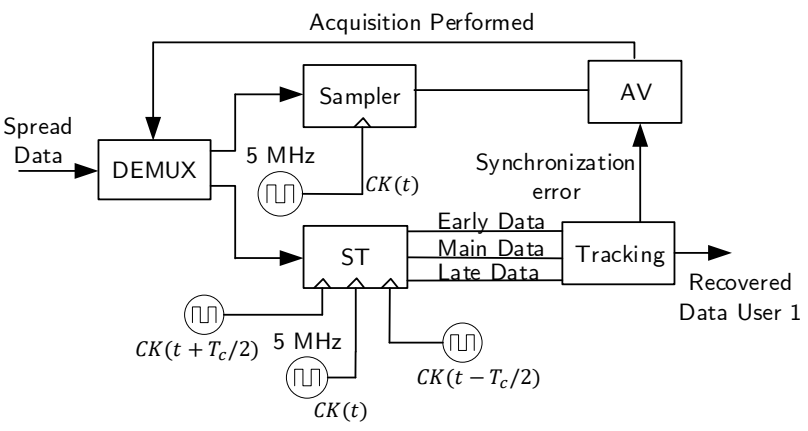

Fig. 5: Conceptual block diagram of AP digital HW based on OCDMA corresponding to one user. Here, AV stands for acquisition and verification. The "ST" block generates early and late replicas of the spread data.

TABLE I: Logic Resources Required for at CN. LUT and and BUFGCTRL stand for look-up table and global clock buffer.

\begin{tabular}{cc}
\hline \hline Logic & Number of Resources \\
\hline LUTs & 95 \\
LUTs as Memory & 22 \\
LUTs as Logic & 73 \\
FFs & 255 \\
BUFGCTRL & 2 \\
\hline
\end{tabular}

the position of ' 1 ' ' in the sequence of the OOC $(49,4,1,1)$ code. We then implemented the full digital HWs described in Section III, on Xilinx ${ }^{\circledR}$ Zynq series 7z020 FPGAs. We have summarized the design synthesis results from the Xilinx ${ }^{\circledR}$ Vivado design suite in Table I. As expected, the Tx remains quite simple to implement, with an estimated on-chip power consumption of $0.752 \mathrm{~W}$. Note that when implemented on an ASIC (Application-Specific Integrated Circuit), the power consumption can be considerably reduced [29].

We have carried out the experimental validation of the proposed O-CDMA system in two steps. First, we verified that the digital HW algorithms were functional, which is described in Subsection IV-A. Then, we experimentally analyzed the performance of the whole system, as explained in Subsection IV-B.

\section{A. Validation of Digital Design}

A design implemented on an FPGA is usually validated functionally by performing a post-implementation simulation on the EDA (Electronic Design Automation) simulator. This, however, does not guarantee the behavior of the design in the real HW. Therefore, a built-in IP (intellectual property) core called integrated logic analyzer (ILA) is used to analyze and debug the system in real time after HW implementation.

Here, both Tx digital HWs are implemented on the same FPGA board, and the Rx digital HW is implemented on another FPGA board. In order to transmit OOC-coded data streams from both Txs with a single FPGA, we simply generated the two streams independently and then added them with an OR gate. This way, on the CN side, the FPGA had a single data output containing the signals from both users. This output was connected with a wire to the input of the second FPGA embedding the AP digital HW. Consequently, the transmission channel between the $\mathrm{CN}$ and the AP could be considered as very low noise and thus not being the source of performance 


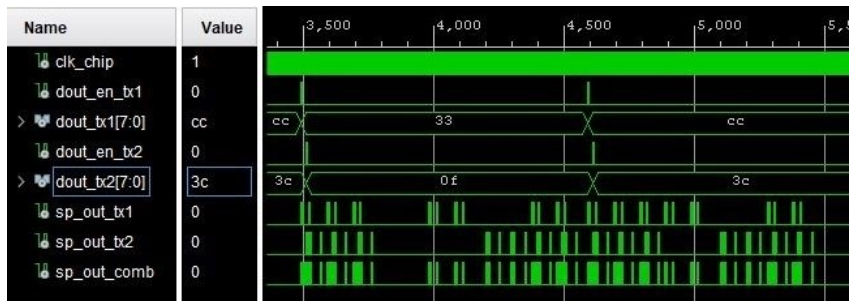

(a) Txs

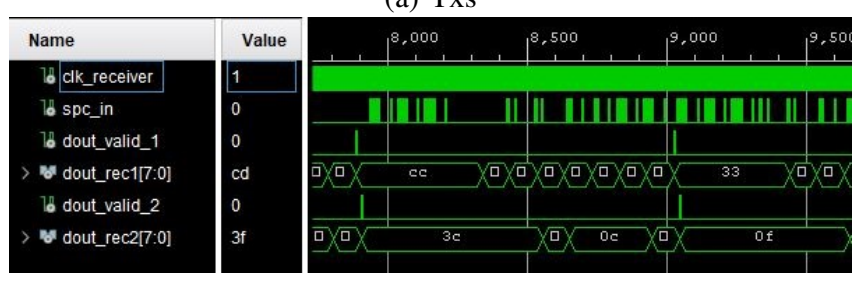

(b) $\mathrm{Rx}$

Fig. 6: Txs and Rx timing diagrams captured through ILA for the case of wired transmission link: (a) Txs send spread data through an OR logic by the port $s p \_o u t \_c o m b$, (b) transmitted data of both Txs are recovered by the Rx.

degradation. This allowed us to evaluate the performance of the digital HW algorithms.

The real-time timing diagrams in Fig. 6 are captured from FPGA's ILA through a JTAG cable. We transferred known pattern of frames from Tx1 (i.e., $33_{16}$ and $\mathrm{cc}_{16}$ ) and Tx2 (i.e., $0 \mathrm{f}_{16}$ and $3 \mathrm{c}_{16}$ ) repeatedly to compare the recovered data after correlation. In Fig. 6(a), the transmitted data sequences from Tx 1 and Tx 2 are denoted by $s p \_o u t \_t x 1$ and $s p \_o u t \_t x 2$, respectively. These spread data generated from Txs are passed through the OR gate and transferred by the port $s p \_o u t \_c o m b$.

The data received by the AP, denoted by $s p c \_i n$, are sampled by a locally generated clock, as seen in Fig. 6(b). The frames recovered by the $\mathrm{Rx}$ are defined as dout_rec1 and dout_rec2, where frames are valid if and only if dout_valid_1 and dout_valid_2 are set to high level for Tx1 and Tx2, respectively. We observed that under the considered "ideal" conditions (i.e., without any noise) the transmitted data bits were recovered from both users without any MAI error.

\section{B. Validation of O-CDMA System}

In order to validate the whole O-CDMA system, we replaced the OR gate and wired channel by the AFEs. Figure 7 shows the experimental setup, where the main parameters are given in Table II. Both Tx AFEs are placed at a distance of $20 \mathrm{~cm}$ from each other and driven by the same FPGA. The measured average transmit power of the IR LED is about $16 \mathrm{~mW}$, that is well bellow the eye-safety requirement [30]. Note that, we have $W=4 \mathrm{ON}$ pulse for the SC length $L=49$.

First, we verified the behavior of the O-CDMA system at short distance. Figure 8 shows the frames recovered at the $\mathrm{Rx}$ when it is placed at a distance of $20 \mathrm{~cm}$ from the Txs. As in Fig. 6(a), we can see that the data being sent by both Txs are recovered completely. Then, we varied the distance $Z$ between the Txs and the Rx from $20 \mathrm{~cm}$ to $1 \mathrm{~m}$ and processed the data captured from the ILA with MATLAB ${ }^{\circledR}$ in order to extract the BER and FER with respect to the distance, as shown in Fig. 9.

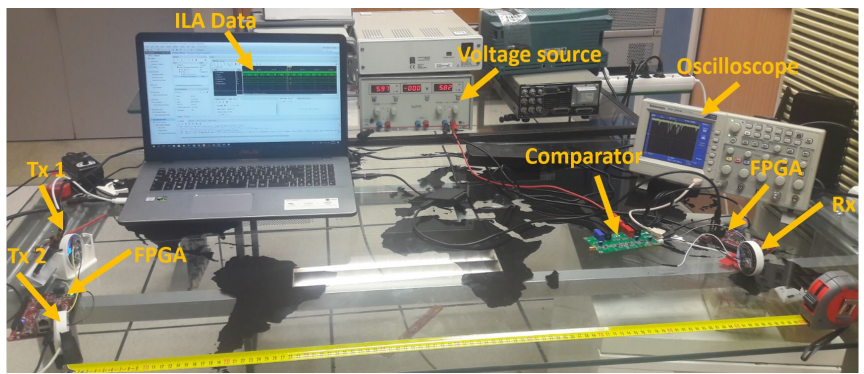

Fig. 7: Experimental setup. Here, Txs are placed at a distance of $1 \mathrm{~m}$ from the Rx.

TABLE II: Parameters Used in the Experiment.

\begin{tabular}{ll}
\hline \hline Parameter & Value \\
\hline OOC code & $(49,4,1,1)$ \\
Number of users & 2 \\
Threshold & 4 \\
CN1 OOC code & $\{0,1,4,14\}$ \\
CN2 OOC code & $\{0,2,7,26\}$ \\
Tx 3 dB modulation BW & $9 \mathrm{MHz}$, \\
Rx 3 dB reception BW & $15 \mathrm{MHz}$, \\
O-CDMA required BW & $5 \mathrm{MHz}$ \\
Rx maximum gain & $62 \mathrm{~dB}$ \\
PD active area & $26.4 \mathrm{~mm}{ }^{2}$ \\
PD responsivity & $0.6 \mathrm{~A} / \mathrm{W}$ \\
Rx full angle at half-sensitivity & $120^{\circ}$ \\
LED wavelength & $940 \mathrm{~nm}$ \\
LED average transmit power & $16 \mathrm{~mW}$ \\
LED semiangle at half power & $60^{\circ}$ \\
\hline
\end{tabular}

We notice that, logically, as the distance $Z$ increases, the BER increases due to decreased received power. Remember from Fig. 2(b) that the comparator acts as a zero-crossing detector, which outputs a $3.2 \mathrm{~V}$ level if the signal is positive, and $0 \mathrm{~V}$ otherwise. Therefore, when the signal is weak and noisy, there might be unexpected zero-crossings leading to an unstable output signal and, eventually, to bit errors.

Moreover, we see from Fig. 9 that the performances of the two Txs are not exactly the same. This is because in our set-up Txs were not exactly at the same distance from the Rx and did not have exactly the same angle of emission with respect to the Rx. This could be considered as a slight form of the near-far problem, which is not mitigated here, as expected for a CHL Rx since we use a comparator instead of a true HL. Indeed, the comparator output is sensitive to the received power level.

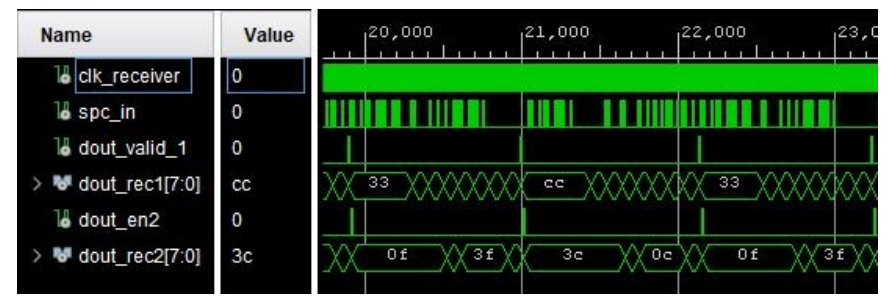

Fig. 8: Recovered data from the two Txs which are positioned at a distance of $20 \mathrm{~cm}$ from the Rx. The data were read through the built-in ILA of FPGA via a JTAG port. 


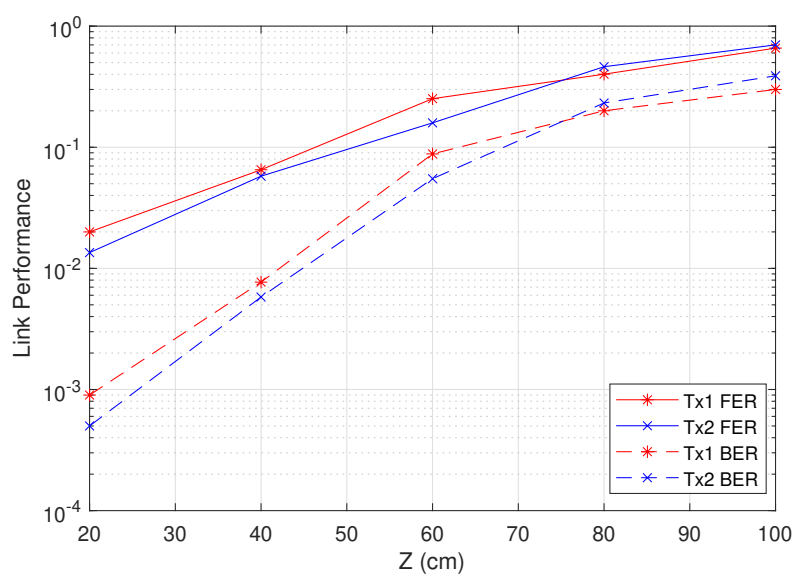

Fig. 9: Measured BER and FER from experiments, where distance $\mathrm{Z}$ between the Txs and the Rx was varied from $20 \mathrm{~cm}$ to $1 \mathrm{~m}$.

\section{CONCLUSIONS}

We proposed digital $\mathrm{HW}$ architectures for the $\mathrm{CN}$ and the AP for the case of extra-WBAN uplinks. The O-CDMA technique was considered to address the multi-user feature of such applications. We also considered AFE design mainly based on CHL while accounting for ease of integration of the AFE part with the digital HW circuitry. Moreover, we implemented the low-complexity digital HW part on the programmable logic. For the case of two users (i.e., two Txs), firstly, we verified the error-free signal reception on a high signal-to-noise ratio (SNR) system (i.e., using a wired link), and then studied the performance of the system with wireless links in terms of BER and FER for different SNR values (i.e., varied link distance). However, the performance of the system can be improved using error-correcting codes. The future direction of this work would be to investigate further the near-far problem and evaluate the robustness of the proposed $\mathrm{Rx}$ architecture.

\section{ACKNOWLEDGMENT}

This work has received funding from the European Union's Horizon 2020 research and innovation programme under the Marie Skłodowska-Curie grant agreement No 764461 (VisIoN).

\section{REFERENCES}

[1] T. Wu, F. Wu, J. Redouté, and M. R. Yuce, "An autonomous wireless body area network implementation towards IoT connected healthcare applications," IEEE Access, vol. 5, pp. 11413-11422, June 2017.

[2] O. Haddad, M. A. Khalighi, S. Zvanovec, and M. Adel, "Channel characterization and modeling for optical wireless body-area networks," in IEEE Open Journal of the Communications Society, 2020, accepted.

[3] O. Haddad and M. A. Khalighi, "Enabling communication technologies for medical wireless body-area networks," in Global LiFi Congress (GLC), June 2019, pp. 1-5, Paris, France.

[4] O. Haddad, M. A. Khalighi, and S. Zvanovec, "Channel characterization for optical extra-WBAN links considering local and global user mobility," in Broadband Access Communication Technologies XIV, B. B. Dingel, K. Tsukamoto, and S. Mikroulis, Eds., vol. 11307, International Society for Optics and Photonics. SPIE, 2020, pp. $89-97$.

[5] S. Movassaghi, M. Abolhasan, J. Lipman, D. Smith, and A. Jamalipour, "Wireless body area networks: A survey," IEEE Communications Surveys \& Tutorials, vol. 16, no. 3, pp. 1658-1686, Mar. 2014.

[6] Z. Ghassemlooy, L. N. Alves, S. Zvanovec, and M. A. Khalighi, Eds., Visible Light Communications: Theory and Applications. CRC-Press, 2017.
[7] N. Barbot, S. Sahuguède, and A. Julien-Vergonjanne, "Performance of a mobile wireless optical CDMA monitoring system," in International Symposium on Wireless Communication Systems (ISWCS), Aug. 2012, pp. 666-670, Paris, France.

[8] L. Chevalier, S. Sahuguède, and A. Julien-Vergonjanne, "Wireless optical technology based body area network for health monitoring application," in IEEE International Conference on Communications (ICC), June 2015 , pp. 2863-2868, London, UK.

[9] M. Patel and J. Wang, "Applications, challenges, and prospective in emerging body area networking technologies," IEEE Wireless Coтmunications, vol. 17, no. 1, pp. 80-88, Feb. 2010.

[10] J. A. Salehi, "Code division multiple-access techniques in optical fiber networks - part I: Fundamental principles," IEEE Transactions on Communications, vol. 37, no. 8, pp. 824-833, Aug. 1989.

[11] S. Zahedi and J. A. Salehi, "Analytical comparison of various fiber-optic CDMA receiver structures," Journal of Lightwave Technology, vol. 18 , no. 12 , pp. $1718-1727$, Dec. 2000.

[12] H. M. H. Shalaby, "Chip-level detection in optical code division multiple access," Journal of Lightwave Technology, vol. 16, no. 6, pp. 1077-1087, June 1998.

[13] S. Zahedi, J. A. Salehi, and M. Nasiri-Kenari, "A photon counting approach to the performance analysis of indoors wireless infrared CDMA networks," in IEEE International Symposium on Personal Indoor and Mobile Radio Communications (PIMRC), vol. 2, Sept. 2000, pp. 928932, London, UK.

[14] S. L. Dhomeja, T. B. Oon, and R. Steele, "Performance of non-directed infrared CDMA," in International Conference on Universal Personal Communications, vol. 1, Oct. 1998, pp. 453-457, Florence, Italy.

[15] K. K. Wong and T. O'Farrell, "Spread spectrum techniques for indoor wireless IR communications," IEEE Wireless Communications, vol. 10, no. 2, pp. 54-63, April 2003.

[16] A. Aminzadeh-Gohari and M. R. Pakravan, "Analysis of power control for indoor wireless infrared CDMA communication," in IEEE International Performance Computing and Communications Conference, Apr. 2006, pp. 297-302, Phoenix, AZ.

[17] B. M. Ghaffari, M. D. Matinfar, and J. A. Salehi, "Wireless optical CDMA LAN: digital design concepts," IEEE Transactions on Communications, vol. 56, no. 12, pp. 2145-2155, Dec. 2008.

[18] B. M. Ghaffari, M. D. Matinfar, and J. A. Salehi, "Wireless optical CDMA LAN: digital implementation analysis," IEEE Journal on Selected Areas in Communications, vol. 27, no. 9, pp. 1676-1686, Dec. 2009

[19] G. C. Yang, "Performance analysis for synchronization and system on CDMA optical fiber networks," IEICE Transactions on Communications, vol. E77B, no. 10, pp. 1238-1248, Oct. 1994.

[20] R. Cameron and B. Woerner, "Performance analysis of CDMA with imperfect power control," IEEE Transactions on Communications, vol. 44, no. 7, pp. 777-781, July 1996.

[21] J. R.Barry, E. A. Lee, and D. G. Messerschmitt, Digital Communications, 3rd ed. Berlin/Heidelberg, Germany: Springer Science \& Business Media, 2012.

[22] F. R. Chung, J. A. Salehi, and V. K. Wei, "Optical orthogonal codes: Design, analysis and applications," IEEE Transactions on Information Theory, vol. 35, no. 3, pp. 595-604, May 1989.

[23] J. A. Salehi and C. A. Brackett, "Code division multiple-access techniques in optical fiber networks - part II: Systems performance analysis," IEEE Transactions on Communications, vol. 37, no. 8, pp. 834-842, Aug. 1989.

[24] S. Mashhadi and J. A. Salehi, "Code-division multiple-access techniques in optical fiber networks - part III: Optical AND logic gate receiver structure with generalized optical orthogonal codes," IEEE Transactions on Communications, vol. 54, no. 8, pp. 1457-1468, Aug. 2006.

[25] J. G. Proakis and M. Salehi, Digital Communications, 5th ed. New York: McGraw-Hill, 2007.

[26] A. Keshavarzian and J. A. Salehi, "Optical orthogonal code acquisition in fiber-optic CDMA systems via the simple serial-search method," IEEE Transactions on Communications, vol. 50, no. 3, pp. 473-483, Mar. 2002.

[27] R. W. Apperson, Z. Yu, M. J. Meeuwsen, T. Mohsenin, and B. M. Baas, "A scalable dual-clock FIFO for data transfers between arbitrary and haltable clock domains," IEEE Transactions on Very Large Scale Integration (VLSI) Systems, vol. 15, no. 10, pp. 1125-1134, Oct. 2007.

[28] T. J. Chaney and C. E. Molnar, "Anomalous behavior of synchronizer and arbiter circuits," IEEE Transactions on Computers, vol. C-22, no. 4, pp. 421-422, Apr. 1973.

[29] I. Kuon and J. Rose, "Measuring the gap between FPGAs and ASICs," IEEE Transactions on Computer-Aided Design of Integrated Circuits and Systems, vol. 26, no. 2, pp. 203-215, 2007.

[30] J. M. Kahn and J. R. Barry, "Wireless infrared communications," Proceedings of the IEEE, vol. 85, no. 2, pp. 265-298, Feb. 1997. 